\title{
Malaria control aimed at the entire population in KwaZulu-Natal negates the need for policies to prevent malaria in pregnancy
}

J M Tsoka-Gwegweni, I Kleinschmidt

Department of Public Health Medicine, Nelson R Mandela School of Medicine, College of Health Sciences, University of KwaZulu-Natal, Durban, and Malaria Research Programme, South African Medical Research Council, Durban

J M Tsoka-Gwegweni, PhD, MPH, MSc, BSc (Hons), BA (Hons)

Tropical Epidemiology Group, Department of Infectious Disease Epidemiology, London School of Hygiene and Tropical Medicine, London, UK, and Malaria Research Programme, South African Medical Research Council, Durban

I Kleinschmidt, $\mathrm{PhD}, \mathrm{MSc}, \mathrm{BSc}$ (Hons), BSc

Corresponding author: JM Tsoka-Gwegweni (tsokagwegweni@ukzn.ac.za)

Background. South Africa has no policy to prevent malaria in pregnancy, despite the adverse effects of the disease in pregnancy. However, malaria control measures consisting of indoor residual spraying and specific antimalarial treatment have been in place since the 1970 s. Information on the burden of malaria in pregnancy in South Africa is needed to indicate whether a specific policy for malaria prevention in pregnancy is necessary.

Objective. To determine the burden of malaria in pregnancy in KwaZulu-Natal (KZN) province, South Africa.

Methods. Pregnant women were enrolled at their first antenatal care visit to three health facilities in Umkhanyakude health district in northern KZN during May 2004 - September 2005 and followed up until delivery. Data collection included demographic details, current and previous malaria infection during pregnancy, haemoglobin concentrations and birth outcomes.

Results. Of the 1406 study participants, more than a quarter were younger than 20 years of age, and more than $90 \%$ were unemployed and unmarried. Although $33.2 \%$ of the women were anaemic, this was not related to malaria. The prevalence and incidence of malaria were very low, and low birth weight was only weakly associated with malaria $(1 / 10)$.

Conclusion. The low burden of malaria in these pregnant women suggests that they have benefited from malaria control strategies in the study area. The implication is that additional measures specific for malaria prevention in pregnancy are not required. However, ongoing monitoring is needed to ensure that malaria prevalence remains low.

S Afr Med J 2013;103(3):172-175. DOI:10.7196/SAMJ.6330 


\section{Malaria in pregnancy}

It is estimated that approximately 50 million pregnant women globally are at risk of contracting malaria each year, and that 10000 mothers and 200000 infants die annually as a result of malaria in pregnancy. ${ }^{[1]}$ Studies conducted in malarious areas of Africa have shown that the burden of malaria in pregnant women is higher than in nonpregnant women. In pregnancy malaria results in anaemia, low birth weight (LBW), prematurity, miscarriage, stillbirth, and perinatal and maternal deaths, ${ }^{[2-4]}$ these complications being particularly severe in pregnant women co-infected with $\mathrm{HIV}^{[5]}$

\section{Control policy for malaria in pregnancy}

The World Health Assembly in 2005 set targets of more than $80 \%$ coverage with all the recommended malaria interventions for pregnant women living in malaria endemic areas. These include indoor residual spraying (IRS) of insecticide for households at risk of malaria. Thirty-three of the 45 African countries that are endemic for malaria had implemented an intermittent preventive treatment of malaria in pregnancy (IPTp) policy by the end of 2006. ${ }^{[1]}$

Given the risks associated with malaria in pregnancy, the World Health Organization (WHO)'s policy for malaria prevention and control in pregnancy recommends IPTp and insecticide-treated bednets (ITNs) as part of the routine antenatal care (ANC) services, and case management of malaria illness. ${ }^{[1,6]}$ IPTp is based on the use of antimalarial drugs given in treatment doses at predefined intervals during pregnancy. The only drug recommended for IPTp is sulfadoxine-pyrimethamine (SP) because of its safety and efficacy during pregnancy and feasibility for use in programmes. The WHO recommends that all pregnant women in endemic areas should be provided with at least two doses of IPT, or three doses in the case of those infected with HIV, beginning in the second trimester. ${ }^{[1,6-7]}$

The second intervention recommended is the use of ITNs, given to pregnant women as early as possible in pregnancy and used up to and after delivery. ITNs are known to reduce human vector contact by physically excluding vector mosquitoes or killing or repelling them if they make contact with the nets. ${ }^{[8]}$ However, the major challenge with implementation of ITN policy is distribution and the development of effective community-based infrastructure to achieve high levels of coverage.

The third method of controlling malaria during pregnancy is prompt treatment of women with symptomatic malaria to prevent fetal loss, preterm delivery and death. The recommended treatment of uncomplicated malaria is artemisinin-combined therapy, because both chloroquine and SP are no longer effective. ${ }^{[1,6]}$ Women should also be screened for anaemia and those with moderate to severe anaemia managed according to the national guidelines for reproductive health

Control policy for malaria in pregnancy in South Africa In South Africa (SA), control for the general population in malarious areas has been provided through vector control measures by using IRS at homesteads since the $1970 \mathrm{~s}{ }^{[9]}$ Protection against malaria is therefore aimed at the entire population and not specifically at pregnant women. While other malaria-endemic countries have implemented IPTp, SA does not have an IPTp policy, mainly because of a lack of information on the burden of malaria in pregnant women. ITNs were used during the 2000/2001 epidemic, but owing to problems of implementation SA does not have a policy on provision of ITNs as a preventive strategy for malaria in pregnancy. The only available national malaria control policy for pregnant women in SA is therefore case management. The revised SA guidelines for the treatment of malaria advise that all pregnant women infected with malaria must be admitted to hospital and treated according to severity of the infection and stage of pregnancy. ${ }^{[9]}$ During the first trimester those diagnosed with uncomplicated malaria are treated with quinine and clindamycin; during the second and third trimesters artemether-lumefantrine treatment may be given. In severe infections intravenous treatment with quinine is recommended during the first trimester and artesunate during the second and third trimesters. ${ }^{[9]}$

SA has no specific policy for prevention of malaria in pregnancy. Implementation of such a policy would require information on the burden of infection in pregnancy. To date this is lacking, particularly for KwaZulu-Natal (KZN) province, which carried $75 \%$ of the burden of malaria until 2007. Notably, a study of the risk of malaria conducted in 1996 in Mpumalanga province found no basis for recommending IPTp for pregnant women, as less than $0.5 \%$ of women were shown to be at risk. ${ }^{[10]}$ This was expected, as the risk of malaria at the time was very low in both Mpumalanga and Limpopo provinces. To better understand the burden of malaria in pregnancy in areas with higher transmission, and to determine whether a prevention policy specifically for malaria in pregnancy was needed, more information was required. This study was therefore initiated to determine the burden of malaria in pregnancy in Umkhanyakude health district in north-eastern KZN, where malaria cases numbered 38543 in 2000 but had decreased to 85 in $2010 .^{[11]}$

\section{Methods}

The study was conducted in Umkhanyakude health district in northeastern KZN. The district adjoins the border with Mozambique, across which there is considerable movement because people on both sides of the border belong to the same clan and many Mozambicans moved south to escape the civil war. Pregnant women were recruited at the ANC clinics of three health facilities (Ndumu Clinic, Manguzi Hospital and Mosvold Hospital), selected because they had reported the highest number of malaria cases in 2003. Malaria transmission in the district is seasonal, peaking in March and April. Every year before the start of the malaria season in November, the inner walls of all houses are sprayed with a residual insecticide. According to the Midyear Populations Estimate for 2011, Umkhanyakude health district has an estimated population of 600000 . More than $90 \%$ of the residents are rural and live in scattered homesteads, some of which are not accessible by road. The population depends on migrant labour, pensions and other social grants, and subsistence farming. Females constitute $56 \%$ of the total population, with $37 \%$ being of reproductive age. ${ }^{[12]}$ Primary healthcare services are provided by district hospitals and fixed and mobile clinics, maternal health services being free. All facilities, including hospital outpatient departments, provide ANC services. The clinics are also used by Mozambican citizens who cross the border to shop and access services.

The study involved a prospective cohort of pregnant women enrolled from May 2004 to September 2005 at their first ANC visit and followed up until delivery. The study sample size was considered to have sufficient statistical power to show a raised risk of malaria infection in pregnant women based on a $10 \%$ annual incidence rate of malaria in the non-pregnant population (KZN Malaria Information System, unpublished data, 2003) and assuming that the relative risk for pregnant women was 1.5 (above 2.0 in other African countries). The sample size and duration were based on the fact that approximately 80 - 100 women presented for ANC at each health facility per month. Trained research assistants and fieldworkers worked with clinic nurses using a data collection sheet designed for this study. At enrolment, written informed consent was obtained from each woman and from parents of teenage mothers. Data were transcribed from ANC clinic attendance cards and the women were interviewed to verify data from the cards. Participants were tested 
using rapid diagnostic tests (ICT Diagnostics, Cape Town) for the presence of Plasmodium falciparum, responsible for $90 \%$ of malaria in SA. ${ }^{[9]}$ Questionnaires captured demographic details and a history of malaria. $\mathrm{Hb}$ was measured using a haemoglobinometer (Hemocue, Johannesburg). Study participants who were positive for malaria were referred to the hospital for treatment. To ensure that cases of malaria that occurred during pregnancy were not missed, women were interviewed and tested for the presence of malaria parasites at least three times during subsequent ANC visits. The data were checked, verified, coded and double-captured into a database. Data were analysed in Epi-Info to produce summary statistics. Further analysis was carried out in Stata (Stata Corporation, Tex.).

The study received ethics approval from the South African Medical Research Council Ethics Review Committee.

\section{Results}

A total of 1406 women aged 12 - 45 years were recruited. The majority had been educated up to secondary school level (Table 1). One quarter were teenagers younger than 20 years. More than $90 \%$ were single and unemployed. More than a third were either primigravidas or multigravidas, while a quarter were secundigravidas. Three-quarters of the women presented for ANC early in the first and second trimesters.

Of the 1406 pregnant women who were tested for malaria, only 1 tested positive, giving a prevalence of $0.07 \%$. Based on a history of malaria (confirmed from clinic charts), 24 of the 1406

\begin{tabular}{|c|c|c|}
\hline Variable & $n$ & $\%$ \\
\hline \multicolumn{3}{|l|}{ Age (years) } \\
\hline$<20$ & 359 & 25.5 \\
\hline $20-29$ & 728 & 51.8 \\
\hline$\geq 30$ & 311 & 22.1 \\
\hline Unknown & 8 & 0.6 \\
\hline \multicolumn{3}{|l|}{ Gravidity } \\
\hline Primigravida & 479 & 34.1 \\
\hline Secundigravida & 408 & 29.0 \\
\hline Multigravida & 512 & 36.4 \\
\hline Unknown & 7 & 0.5 \\
\hline \multicolumn{3}{|l|}{ Schooling } \\
\hline None & 246 & 17.5 \\
\hline Primary & 293 & 20.8 \\
\hline Secondary & 857 & 61.0 \\
\hline Unknown & 10 & 0.7 \\
\hline \multicolumn{3}{|c|}{ Antenatal care attendance } \\
\hline Early & 1055 & 75.1 \\
\hline Late & 319 & 22.7 \\
\hline Unknown & 32 & 2.3 \\
\hline \multicolumn{3}{|l|}{ Marital status } \\
\hline Single & 1291 & 91.8 \\
\hline Married & 79 & 5.6 \\
\hline Unknown & 36 & 2.6 \\
\hline \multicolumn{3}{|l|}{ Employed } \\
\hline Yes & 82 & 5.8 \\
\hline No & 1321 & 94.0 \\
\hline
\end{tabular}

Table 2. Malaria, anaemia and birth outcomes

\begin{tabular}{|c|c|c|c|c|c|c|}
\hline \multirow[b]{2}{*}{ Outcome } & \multicolumn{3}{|c|}{ Malaria-positive } & \multicolumn{3}{|c|}{ Malaria-negative } \\
\hline & $N$ & $n$ & $\%$ & $N$ & $n$ & $\%$ \\
\hline Anaemia $(33.2 \%)$ & 18 & 2 & 11 & 1030 & 346 & 33.6 \\
\hline LBW (8.1\%) & 10 & 1 & 10 & 642 & 52 & 8 \\
\hline Preterm (1.2\%) & 10 & 0 & 0 & 642 & 8 & 1.25 \\
\hline Stillbirth (0.6\%) & 10 & 0 & 0 & 642 & 4 & 0.6 \\
\hline IUGR (0.15\%) & 10 & 0 & 0 & 642 & 1 & 0.16 \\
\hline
\end{tabular}

women reported that they had had malaria during the current pregnancy. This translated into a malaria incidence of 23 cases per 1000 person years, allowing for follow-up until delivery (approximately 9 months). Of the 1048 study participants whose $\mathrm{Hb}$ was measured, $33.2 \%$ were anaemic $(\mathrm{Hb}<11 \mathrm{~g} / \mathrm{dl})$ and $1.4 \%$ had moderate to severe anaemia $(\mathrm{Hb}<8 \mathrm{~g} / \mathrm{dl})$. The prevalence of anaemia was lower in the women who reported having been treated for malaria during this pregnancy than in women who had not had malaria (Table 2). Birth outcomes were recorded for 652 deliveries that occurred at the study sites. Birth records from outside the study area were not available, with most unrecorded deliveries taking place at home or in facilities in Mozambique. Information on study participants who had home deliveries and miscarriages was not available at the health facilities studied. None of the birth outcomes were associated with malaria apart from LBW, but evidence for this association was weak (Table 2).

\section{Discussion}

The study sample was characterised by high rates of teenage pregnancy, unemployment and unmarried status. These have remained unchanged over the past 5 - 10 years, except that the rate of teenage pregnancy has almost doubled since $1998{ }^{[13]}$ in keeping with current prevalence of teenage pregnancy in SA of more than $60 \% \cdot{ }^{[14]}$ This is of concern given that the prevalence of HIV among the pregnant population in KZN is more than $40 \% .{ }^{[15]}$

The findings show that the majority of the study participants attended for ANC early in their pregnancy. This is important for the implementation of beneficial interventions such as the prevention of mother-to-child transmission (PMTCT) of HIV, IPTp, ITNs and other programmes aimed at improving pregnancy outcomes. Moreover, the relatively high level of education among the study participants suggests that health education messages would reach the majority of women in the course of their accessing health services. ${ }^{[13]}$

Both the incidence and prevalence of malaria in pregnancy were found to be very low. This might have been expected, since the burden of malaria in the general population has been dramatically reduced as a result of policy changes in malaria control initiatives in KZN and throughout SA. ${ }^{[1]]}$ The findings are in agreement with research in other countries indicating that malaria in pregnancy is associated with $\mathrm{LBW}^{[2-4]}$ although the numbers in this study were too small to provide decisive confirmation. There was no evidence of an association of malaria with anaemia, preterm delivery, stillbirth or intra-uterine growth retardation. Compared with national surveys and previous research conducted in the study area, the prevalence of LBW remains high and unchanged, ${ }^{[16]}$ suggesting that efforts aimed at PMTCT of HIV are having little impact on LBW. Although the numbers were too low to show evidence of an association, preterm delivery and stillbirth appear to be linked to anaemia. 


\section{RESEARCH}

\section{Conclusions}

The low prevalence and incidence of malaria suggest that: $(i)$ the high level of anaemia was not attributable to malaria and requires further investigation; (ii) as houses in the study area were sprayed and cases of malaria treated with effective drugs, pregnant women are likely to have benefited from malaria control efforts aimed at the general population; (iii) in the face of the high level of HIV in the province, a well-run malaria control programme that reduces the risk of malaria infection also lowers the risk of co-infection and its attendant complications, particularly in pregnancy; and (iv) there is no basis on which to recommend measures specifically aimed at prevention of malaria in pregnancy in $\mathrm{KZN}$, as the highly successful vector control programme, aimed at the entire population, appears to be sufficient.

This study is the first to focus on malaria in pregnancy and to determine the effects of malaria on birth outcomes in SA, particularly in the high-risk province of KZN. At the time the study was carried out, the malaria endemic districts of the province accounted for $75 \%$ of the burden of malaria in SA. Limpopo province has however, become the highest malaria risk province and during the 2010 malaria transmission season reported 4174 cases compared with 2187 and 380 in Mpumalanga and KZN, respectively. ${ }^{[11]}$ Future research should therefore focus on malaria in pregnancy in Limpopo. The findings from this study indicate that broader prevention strategies are sufficient to reduce the risk to pregnant women. This has policy implications for SA and other countries for the prevention of malaria in pregnancy.

Acknowledgements. This project was jointly funded by the South African Medical Research Council and the World Health Organization, AFRO. The authors are also grateful to the collaborators, management of health facilities and communities in Umkhanyakude district, the Department of Health, and the research assistants, fieldworkers and staff in the Database Section at the Malaria Research Programme of the South African Medical Research Council.

\section{References}

1. World Health Organization/GMP. Global Fund Proposal Development: WHO Policy Brief on Malaria. Geneva: WHO, 2011 .

2. Ndyomugyenyi R, Magnussen P. Anaemia in pregnancy: Plasmodium falciparum infection is an mportant cause in primigravidae in Hoima district, western Uganda. Ann Trop Med Parasito

3. Shulman C. Malaria in Pregnancy. Roll Back Malaria Infosheet 4. 2001-2010. United Nations Decade to Roll Back Malaria. Geneva: World Health Organization, 2002.

4. Steketee RW, Nahlen BL, Parise ME, Menendez C. The burden of malaria in pregnancy in malariaendemic areas. Am J Trop Med Hyg 2001;64(1-2 Suppl):28-35.

5orld Health Organization/Department of HIV/AIDS/RBM. Malaria and HIV Interactions and their Implications for Public Health Policy. Report of a Technical Consultation, $23-25$ June 2004. Geneva: World Health Organization, 2005

6. World Health Organization. A Strategic Framework for Malaria Prevention and Control During Pregnancy in the African Region. Brazzaville: WHO, 2004.

7. World Health Organization. World Malaria Report 2010. Geneva: WHO, 2010.

8. Lengeler, C. Insecticide-treated bed nets and curtains for preventing malaria. Cochrane Database of Systematic Reviews, CD0003632004.

9. Department of Health. Guidelines for Treatment of Malaria in South Africa. Pretoria: Departmen of Health, 2010.

10. Govere J, Billinghurst K, Durrheim DN, Hansford CF. Malaria risk during pregnancy in Mpumalanga province, South Africa. S Afr Med J 1999;89(6):625.

11. Moonasar D, Nutulaganti T, Kruger PS, et al. Malaria control in South Africa 2000-2010: beyond MDG6. Malar J 2012;11:294. [http://dx.doi.org/10.1186/1475-2875-11-294].

12. Statistics South Africa. Midyear Population Estimates 2011. Statistical release PO302. Pretoria StatsSA, 2011.

13. Tsoka JM, le Sueur D, Sharp BL. Maternal health service utilisation in Ubombo district. South African Journal of Obstetrics and Gynaecology 2003;93(3):70-74.

14. Shisana O, Rehle T, Simbayi LC, et al., the SABSSM III Implementation Team. South African Nationa HIV Prevalence, Incidence, Behaviour and Communication Survey 2008: A Turning Tide Among Teenagers? Cape Town: HSRC Press, 2009.

15. Department of Health. National Antenatal Sentinel HIV and Syphilis Prevalence Survey in South Africa, 2009. Pretoria: Department of Health, 2010.

16. Pattinson RC, ed. Saving Babies 2006-2007: Sixth Perinatal Care Survey of South Africa. Pretoria: Tshepesa Press, 2009.

Accepted 22 November 2012 